\title{
The Generalized Likelihood Ratio Test and the Sparse Representations Approach
}

\author{
Jean Jacques Fuchs \\ IRISA, Univ. de Rennes 1, Campus de Beaulieu, 35042 Rennes Cedex, France \\ fuchs@irisa.fr
}

\begin{abstract}
When sparse representation techniques are used to tentatively recover the true sparse underlying model hidden in an observation vector, they can be seen as solving a joint detection and estimation problem. We consider the $\ell_{2}-\ell_{1}$ regularized criterion, that is probably the most used in the sparse representation community, and show that, from a detection point of view, minimizing this criterion is similar to applying the Generalized Likelihood Ratio Test. More specifically tuning the regularization parameter in the criterion amounts to set the threshold in the Generalized Likelihood Ratio Test.
\end{abstract}

\section{Introduction}

Sparse representation techniques can be considered to have two main applications. The first and direct one [1] being compression i.e. given a data vector represent it in a sparse way, which is then useful in coding, compression, archiving, etc. The second and less direct one being the retrieval of a true sparse representation from noisy observations [2]. The application in statistics [3], in a variable selection context, when the number of potential instruments or predictor variables is larger than the number of observations, can be seen as being somehow in between these two main classes of applications. One indeed seeks a sparse representation on a set of say physically meaningful instruments though strictly speaking there is no prior true sparse representation.

The second application is the one considered in this paper. It has a long history in the signal processing community and has started well before the present sparse representations era in a number of different domains such as identification, source separation, model fitting, inverse problems among others, 45.

In the next section, one shortly describes the general sparse representation problem that will be considered. It potentially covers a large class of the applications listed above. The classical problem of the detection of signals in noise of known covariance is then presented and the generalized likelihood ratio test (GLRT) developed [67]. It is then shown that setting the value of the threshold in the GLRT is equivalent to tuning the hyper-parameter in the $\ell_{2}-\ell_{1}$ regularized criterion used to get a sparse representation. Finally a specific application is analysed and some simulation results presented.

A. Elmoataz et al. (Eds.): ICISP 2010, LNCS 6134, pp. 245 253, 2010.

(C) Springer-Verlag Berlin Heidelberg 2010 


\section{Sparse Representations in an Estimation Context}

Basically and without seeking generality to keep the model simple [8, sparse representations techniques can, for instance, be applied as soon as an n-dimensional observation vector $b$ is the sum of an unknown number $P$ of weighted contributions $a(\theta)$ in additive white noise, i.e.,

$$
b=\sum_{k=1}^{P} \alpha_{k} a\left(\theta_{k}\right)+e
$$

with $e \in N\left(0, \sigma^{2} I_{n}\right)$ and $a(\theta)$ a parametrized vector function depending upon a scalar $\theta \in[0,1]$. To both decide upon the number $P$ of contributors and to estimate theirs characteristics $\left\{\alpha_{k}, \theta_{k}\right\}$, one then solves

$$
\min _{x} \frac{1}{2}\|A x-b\|_{2}^{2}+h\|x\|_{1} \quad \text { with } h>0,
$$

with $\|x\|_{1}=\sum_{k}\left|x_{k}\right|$ and $\|x\|_{2}^{2}=\sum_{k}\left|x_{k}\right|^{2}$ and $A$ an $n \times m$ matrix $(m \gg n)$ with column vectors $a_{j}=a(j / m) /\|a(j / m)\|_{2}, \quad 1 \leq j \leq m$ with $m \gg n$, the normalized $a(\theta)$-vector on a uniform grid.

One seeks to reconstruct the observation vector $b$ by a model of the form $A x$ where $x$ is a high-dimensional but sparse (having just a few non-zero components) vector that indicates which columns in $A$ are useful in the construction of a approximation of $b$. The sparseness is ensured by the presence of the $\ell_{1}$ norm in the criterion. Ideally, there are between $P$ and $2 P$ non-zero components in the optimal $x$.

This optimization problem being convex 9 ] has generically a unique optimum. One deduces from its optimum both an estimate $\hat{P}$ of $P$ and the estimates of $\left\{\alpha_{k}, \theta_{k}\right\}$ for $\mathrm{k}=1$ to $\hat{P}$. The parameter $h$ which appears in (2) has to be fixed by the user with care since it plays an important role that we do emphasize below.

\section{Detection of Signals in Noise}

In the context of model (1), the associated basic detection problem is known as the detection of signals in noise of known covariance. It is well documented and in its elementary version it consists in deciding between

$$
H_{0}: \quad b=e \quad \text { and } \quad H_{1}: \quad b=\alpha a\left(\theta_{o}\right)+e
$$

with $\theta_{o}$ known, i.e., to decide whether $\alpha=0$ or $\alpha \neq 0$. One way to take the decision is then to use the likelihood ratio test (LRT) which in this case amounts to compare

$$
\frac{1}{\sigma^{2}} \frac{\left(a\left(\theta_{o}\right)^{T} b\right)^{2}}{\left\|a\left(\theta_{o}\right)\right\|_{2}^{2}}
$$

to a threshold. The quantity to be tested is clearly linked to the so-called matched filter output 10] $\left(a\left(\theta_{o}\right)^{T} b\right) /\left\|a\left(\theta_{o}\right)\right\|_{2}$. This test procedure is optimal in the sense that it maximizes the detection probability for a given probability of false alarm. 
In the more general case where $\theta_{o}$ is also unknown, a suboptimal strategy known as the generalized likelihood ratio test (GLRT) 6.7] can be used. It is a quite general approach to detection of signals that include unknown parameters. The GLR is defined as the ratio of the maximum value of the likelihood under $H_{1}$ to the maximum value under $H_{0}$, the maximum being with respect to the unknown parameters. It is shown below that it amounts to project the observation $b$ onto the manifold associated with $a(\theta)$ for $\theta \in[0,1]$ and to retain the model leading to the projection with largest modulus.

To confirm these observations let us define the GLRT statistic $t(b)$

$$
t(b)=\max _{\alpha, \theta}\left\{2 \log f_{1}(b)\right\}-2 \log f_{0}(b)
$$

with $f_{0}(b)$ and $f_{1}(b)$ the probability density functions (p.d.f.) of $b$ under $H_{0}$ and $H_{1}$, respectively. One has, under $H_{1}$

$$
2 \log f_{1}(b)=-n \log \left(2 \pi \sigma^{2}\right)-\frac{1}{\sigma^{2}}\|b-\alpha a(\theta)\|_{2}^{2}
$$

Its optimum w.r.t. $\alpha$ is attained at:

$$
\hat{\alpha}=\frac{b^{T} a(\theta)}{a(\theta)^{T} a(\theta)}
$$

and by substitution of this value, its optimum over $\theta$ satisfies

$$
\hat{\theta}=\arg \min _{\theta} b^{T}\left(I-\frac{a(\theta) a\left(\theta^{T}\right.}{a(\theta)^{T} a(\hat{\theta})}\right) b=\arg \max _{\theta} \frac{\left(b^{T} a(\theta)\right)^{2}}{a(\theta)^{T} a(\hat{\theta})} .
$$

The associated value of the loglikelihood is then

$$
\max _{\alpha, \theta}\left\{2 \log f_{1}(b)\right\}=-n \log \left(2 \pi \sigma^{2}\right)-\frac{1}{\sigma^{2}}\left(\|b\|_{2}^{2}-\max _{\theta} \frac{\left(b^{T} a(\theta)\right)^{2}}{a(\theta)^{T} a(\hat{\theta})}\right) .
$$

Since under $H_{0}$, the value of loglikelihood is simply $2 \log f_{0}(b)=-n \log \left(2 \pi \sigma^{2}\right)-$ $\left(1 / \sigma^{2}\right)\|b\|_{2}^{2}$, the statistic $t(b)$ in (44) is now fully characterized and the GLRT procedure is thus to compare it to a threshold $\eta$

$$
t(b)=\frac{1}{\sigma^{2}} \max _{\theta} \frac{\left(b^{T} a(\theta)\right)^{2}}{a(\theta)^{T} a(\theta)}><\eta .
$$

and to decide $H_{1}$ if the maximum is greater than the threshold and $H_{0}$ otherwise.

The threshold $\eta$ is generally fixed so as to make the probability of false alarm equal to a user-chosen value $P_{F A}$. To fix the threshold, one thus needs to find the p.d.f. of $t(b)$ when $b=e$, or equivalently the p.d.f. of

$$
t(w)=\max _{\theta}\left(w^{T} a(\theta)\right)^{2} /\|a(\theta)\|^{2} \quad \text { with } \quad w=N\left(0, I_{n}\right) .
$$

Seeking the probability density function of the maximum of a set or a continuum of random variables is in general a difficult problem [11|12 when the variables are not independent. 


\section{Detection Properties of the Sparse Representations Approach}

Let us establish that, if one wants to decide between the two hypothesis in (3), comparing the GLR to a threshold $\eta$, is equivalent to comparing the optimum $x$ of (2) to the zero vector. To prove this point, let us introduce the conditions satisfied by the optimum of the criterion (2). This criterion is convex [9] and the necessary and sufficient condition for $x$ to be a global minimum of (2) is that the vector zero is a sub-gradient of the criterion at $x$ :

$$
\exists u \in \partial\|x\|_{1} \quad \text { such that } A^{T}(A x-b)+h u=0,
$$

where $u$ is a sub-gradient of $\|x\|_{1}$, i.e., a vector belonging to the set called the sub-differential of $\|x\|_{1}$, denoted $\partial\|x\|_{1}$, and satisfying

$$
\begin{aligned}
& \partial\|x\|_{1}=\left\{u \mid u^{T} x=\|x\|_{1},\|u\|_{\infty} \leq 1\right\} \\
& =\left\{u \mid u_{i}=\operatorname{sign}\left(x_{i}\right) \text { if } x_{i} \neq 0 \text { and }\left|u_{i}\right| \leq 1 \text { otherwise }\right\} .
\end{aligned}
$$

To prove that if:

$$
h \geq\left\|A^{T} b\right\|_{\infty}=\max _{k}\left|a_{k}^{T} b\right|
$$

the optimal $x$ is at zero, one simply checks that $\left\{x=0, u=(-1 / h) A^{T} b\right\}$ satisfies (7) with the given requirements on $u$ in $\partial\|x\|_{1}$. Intuitively this means that in (2) and a large $h$ satisfying (8), no component in $x$ is worth being made non-zero.

Since $a_{k}$ in (8) is equal to $a(k / m) /\|a(k / m)\|_{2}$, this establishes the announced equivalence, since to tune $h$ one now needs to evaluate the p.d.f. of

$$
\max _{k} \frac{\left|w^{T} a(k / m)\right|}{\|a(k / m)\|} \quad \text { with } \quad w=N\left(0, I_{n}\right) .
$$

compare with (6). The sole difference is that in (8) the maximum is sought over $m$ values of $\theta$, while in the GLRT it is sought over a continuum of values of $\theta$.

As a matter of fact, if a unique component in the optimal $x$ of (2) is non-zero, say the i-th component $x_{i}$ then it can be deduced from (7), that its value is given by $[13]$ :

$$
x_{i}=a_{i}^{T} b-h \operatorname{sign}\left(x_{i}\right),
$$

a sum of two terms, where the first term is the optimal (ML) amplitude estimate, see (5), and the second term is a bias due to the presence of the penalization $h\|x\|_{1}$ in (2) that systematically diminishes the absolute value by $h$.

The sparse representation approach continues to work if more than just one signal is present and can be seen as an extension of the GLRT procedure applicable to a generalization of (3). The presence of the systematic bias on the amplitude estimates is well known, it has then a more intricate expression than above, but remains easy to remove. 


\section{Typical Application: A Sum of Sinusoids in White Noise}

\subsection{The Model}

Let us apply the observations made above to the specific sinusoid-plus-noise example, where the parameter $\theta$ to be estimated is two-dimensional

$$
b_{j}=s_{j}+e_{j}, \quad \text { with } s_{j}=\sum_{k=1}^{P} \alpha_{k} \cos \left(\omega_{k} j+\varphi_{k}\right), \quad \text { with } \quad j \in\{0, n-1\}
$$

where $e_{j}$ are white Gaussian noise samples. To cast this application into the form required by the sparse representations approach in (2), one switches to complex weights $x=\rho e^{i \varphi}$ and complex basis vector denoted $c(\omega)$ corresponding to cisoids with equispaced pulsations $\omega_{k}=2 \pi(k / m)$ with $k \in\{0, m-1\}$ :

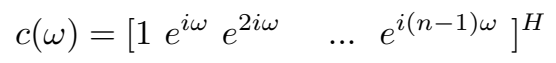

Another possibility [15] is to store in the columns of $A$, real vectors $a(\omega, \varphi)$ where $\omega$ takes takes $m_{\omega}$ equispaced values in $\left[0, \pi\left[\right.\right.$ and $\varphi$ takes $m_{\varphi}$ values equispaced in $[0, \pi]$ but the complex representationis preferred in the sequel.

The component $(q, p)$ of $A$ is thus equal to $a_{q, p}=\exp (-2 i \pi(q-1)(p-1) / m)$, i.e. the $q-t h$ component of the column of the cisoid $c(\omega)$ for $\omega=2 \pi(p-1) / m)$. To fix ideas the number $m$ of columns in $A$ is taken even, it follows that $a_{k}=$ $\bar{a}_{m+2-k}$, i.e. the columns in $A$ are pairwise complex conjugate. Similarly, for the reconstructed vector $A x$ to be a real vector like the observation vector $b$ it aims to reconstruct, the same hermitian symmetry property will hold for $x$, i.e. $x_{k}=\bar{x}_{m+2-k}$ for $k=2$ to $m / 2$. Indeed one can establish by contradiction that the optimal solution $x$ of (2) will always satisfy this property. It follows then from (8) that when $b$ is composed of a single sinusoid, it will be detected by the sparse representations approach if

$$
h<\left\|A^{H} b\right\|_{\infty}=\max _{k}\left|a_{k}^{H} b\right| .
$$

\subsection{The Associated GLRT}

One considers the single sinusoid case, see (3), where one decides if a single sinusoid with unknown pulsation and initial phase is present or not present. For the GLRT approach and under $H_{1}$, one then has to find the MLE estimates of the different quantities. One establishes below that the ML estimate of the pulsation corresponds to the location of the maximum of the periodogram, just like in the sparse representation approach, see (11).

With $a(\omega, \varphi)$ a n-dimensional vector whose $k$-th component is $\cos ((k-1) \omega+\varphi)$, the probability density function of $b$ is

$$
f_{\alpha, \omega, \varphi}(b)=\left(2 \pi \sigma^{2}\right)^{-n / 2} \exp \left(-\left(1 / 2 \sigma^{2}\right)\|b-\alpha a(\omega, \varphi)\|_{2}^{2}\right) .
$$


Under $H_{0}, \alpha=0$ and using the notations of the previous section

$$
-(2 / n) \log f_{0}(b)=\log (2 \pi)+\log \sigma^{2}+\left(1 / n \sigma^{2}\right)\|b\|^{2} .
$$

Under $H_{1}$, one seeks the maximum likelihood estimates (MLE) of the unknowns, $\alpha, \omega$, and $\varphi$, they are obtained by locating the maximum of $f_{\alpha, \omega, \varphi}(b)$. Equating to zero the partial derivatives of $f$ with respect to $\alpha$ yields

$$
\hat{\alpha}=\left[a(\omega, \varphi)^{T} b\right] /\left[a(\omega, \varphi)^{T} a(\omega, \varphi)\right]
$$

Substitution of this values into $f$, one observes that maximizing this function with respect to $\omega$ and $\varphi$ amounts to maximize

$$
\max _{\omega, \varphi}\left(b^{T} a(\omega, \varphi)\right)^{2} /\|a(\omega, \varphi)\|^{2} .
$$

If one now assume that the norm of $a(\omega, \varphi)$ is independent of $\omega$ and $\varphi$, which is realistic [16] for large $n$ or $\omega$ not too close to 0 or $\pi$, the sought-for estimates of $\omega$ and $\varphi$ are obtained as

$$
\hat{\omega}, \hat{\varphi}=\arg \max _{\omega, \varphi} g(\omega, \varphi) \quad \text { with } g(\omega, \varphi)=\left|b^{T} a(\omega, \varphi)\right| .
$$

Let us denote $c(\omega, \varphi)$ the vector filled with complex exponentials whose real part is $a(\omega, \varphi)$ and $B(\omega)$, the discrete Fourier transform (DFT) of $b$ with modulus $\rho(\omega)$ and phase $\theta(\omega)$. One then has

$$
\begin{aligned}
g(\omega, \varphi)=\left|b^{T} a(\omega, \varphi)\right| & =\left|\Re\left(b^{T} c(\omega, \varphi)\right)\right|=\left|\Re\left(b^{T} c(\omega, 0) \exp (-i \varphi)\right)\right| \\
& =|\Re(B(\omega) \exp (-i \varphi))|=\rho(\omega)|\cos (\theta(\omega)-\varphi)| .
\end{aligned}
$$

The maximum of this last expression is attained at $\hat{\omega}$ the value of $\omega$ that maximizes $\rho($.$) and then at \hat{\varphi}=\theta(\hat{\omega})$ to make the cosine equal to one. This means that the MLE of $\omega$ is, for large $n$, the values of $\omega$ for which the modulus $\rho(\omega)$ of the DFT of $b$ is maximal. The other estimates are then obtained by substitution of this estimate $\hat{\omega}$, one first obtains $\hat{\varphi}$ and then $\hat{\alpha}$.

Since

$$
\max _{\omega, \varphi}\left|b^{T} a(\omega, \varphi)\right|=\max _{\omega} \rho(\omega)=\max _{\omega}\left|b^{T} c(\omega)\right|
$$

with $c(\omega)=c(\omega, 0)$ the column associated with the cisoid at pulsation $\omega$, it follows that the GLR statistic (44) can be taken as

$$
t(b)=\left(1 / \sigma^{2}\right) \max _{\omega}\left|b^{T} c(\omega)\right|^{2}
$$

which is nothing but, up to a multiplicative constant, the maximum of the periodogram and is also the test strategy performed by the sparse representations approach, compare with (8), since $a_{k}$ the generic notation for the $k$-th column of the $A$ matrix is now precisely $c(\omega)$ with $\omega=2 \pi(k-1) / m$. 


\subsection{Tuning the Parameter $h$}

The simple scenario described in (3) is considered and the parameter $h$ is set $h$ to achieve a given probability of false alarm. A false alarm appears when the algorithm decides that there is (at least) one sinusoid present in the observation vector $b$ while indeed there is none, i.e. while $b=e$. From Section 4 and relation (11), it follows that this is the case if $\left\|A^{H} e\right\|_{\infty}>h$, where $a_{k}$ is the k-th (complex) column vector of the matrix $A$ and $\mathrm{e}=N\left(0, \sigma_{e}^{2} I_{n}\right)$. The vector $A^{H} e$ is a vector of $m$ scalar complex random dependent variables build upon the $n$ independent real Gaussian variables present in $e$. Each of these complex random variables is of the form $c^{H} e$ with $c$ an n-dimensional complex vector (10), with norm $\sqrt{n}$, that can be decomposed into the sum of its real part (cosines) and imaginary part (sines) so that $c^{H} e=v_{\cos }^{T} e-i v_{\sin }^{T} e$ where the two vectors $v_{\sin }$ and $v_{\cos }$ are quasi-orthogonal and of similar norm.

It follows that $\sqrt{2 /\left(n \sigma_{e}^{2}\right)} c^{H} e$ is, approximatively, a complex Gaussian random variable whose real and imaginary parts are Gaussian, independent, zero mean and with unit variance and thus that $\left(2 /\left(n \sigma_{e}^{2}\right)\right)\left|c^{H} \mathbf{e}\right|^{2}$ is, approximatively, a Chi-square random variable with 2 degrees of freedom.

Let us now recall a quite standard result. For $n$ independent and identically distributed random variables, say $w_{i}$, the following result holds

$$
\begin{gathered}
P\left(\max _{i \in(1, n)} w_{i}>t\right)=p \Leftrightarrow P\left(\max _{i \in(1, n)} w_{i}<t\right)=1-p \\
\Leftrightarrow P\left(w_{i}<t\right)^{n}=1-p \Leftrightarrow P\left(w_{i}>t\right)=1-(1-p)^{(1 / n)} \simeq \frac{p}{n},
\end{gathered}
$$

where $P\left(w_{i}<t\right)$ denotes the probability of the random variable $w_{i}$ to be smaller than $t$ and where the approximation in the last step is valid for small probability $p$ and large $n$.

In the present situation, one can consider that since the $m$ dependent variables in $A^{H} e$ depend only upon the $n$ degrees of freedom in $e$, there are only $n$ independent random variables that are $\chi_{2}^{2}$. Applying the previous result, then leads to

$$
h=\sigma_{e} \sqrt{\frac{n}{2}} \sqrt{t}, \text { with } \operatorname{Pr}\left(\chi_{2}^{2}>t\right)=\frac{p}{n} .
$$

A precise analysis is extremely difficult and no analytical expression that would allow to fix $h$ exactly exists to our knowledge 111214. The simulations below (see Section [5.4) show that this approximate way to fix $h$ is quite accurate.

\subsection{Simulation Results}

To check the validity of the analysis performed in Section 5.3. we evaluate the probability of false alarm of GMF. We consider $n=100$ data points and $m=$ 1000 columns in $A$.

For a probability of false alarm of 10 percent, i.e., $p=.1$ the value of $h$ given by (12), is $h=26.28$ and 10000 independent realizations, of noise only observations $b=e$ were simulated. The procedure detected wrongly a sinusoid (had a optimal $z$ not identically zero) for 1348 among the 10000, i.e. 13 percent. 
As a matter of fact, if we estimate for each realization, the actual noise variance and use it to fix the value of $h$ accordingly using (12), the number of false alarms, for the same 10000 realizations, dropped to 1077, quite close to the expected 10 percent.

This means that the approximate evaluation of the statistical properties of the moduli of the outputs of the Fourier Transform under $H_{0}$ gives a threshold that is quite accurate and that the choice of the threshold is quite sensitive for this specific detection problem.

Let us now simulate a single sinusoid with $\alpha=\sqrt{2}$ and the same unit variance noise. This corresponds to a signal to noise ratio (SNR) of $0 \mathrm{~dB}$. For $h$ given by (12), the sparse representations approach detects systematically the sinusoid and sometimes detects a second sinusoid. The results obtained over 10000 independent realizations indicate that the proposed procedure attains the Cramer-Rao bounds [16] for the true sinusoid and that among the 10000 realizations, a second sinusoid (by far much weaker) is detected in 1259 realizations, i.e. in again about 13 percent of the realization, as expected since the true sinusoid is strong enough to be always detected.

\section{$6 \quad$ Concluding Remarks}

It has been shown that in a detection and estimation context, the basic sparse representations criterion

$$
\min _{x} \frac{1}{2}\|A x-b\|_{2}^{2}+h\|x\|_{1} \quad \text { with } h>0,
$$

acts as a generalized likelihood ratio test (GLRT) and that the tuning of the hyperparameter $h$ in this criterion relies on the same statistical analysis then the tuning of the threshold in the GLRT. Since this criterion is equivalent to

$$
\min _{x}\|x\|_{1} \quad \text { subject to } \quad\|A x-b\|_{2}^{2} \leq \rho,
$$

one could argue that, since tuning $\rho$ is much easier, this second criterion should be preferred. But this is of course wrong. From a detection point of view, the second criterion is indeed equivalent to a energy detector with much poorer probability of detection for a given probability of false alarm, than the GLRT. The relation between $h$ and $\rho$ which makes these criteria equivalent is indeed unknown a priori and data dependent and there is no way to exploit this relation to bypass the sophisticated analysis needed to tune $h$.

\section{References}

1. Donoho, D.: Compresssed sensing. IEEE-T-IT 52, 1289-1306 (2006)

2. Bofil, P., Zibulevski, M.: Underdetermined blind source separation using sparse representations. In: Signal Processing, November 2001, vol. 81, pp. 2353-2362. Elsevier, Amsterdam (2001) 
3. Tibshirani, R.: Regression shrinkage and selection via the Lasso. J. Royal Statist., Soc. B 58(1), 267-288 (1996)

4. Kwakernaak, H.: Estimation of pulse heights and arrival times. Automatica 16, 367-377 (1980)

5. Fuchs, J.J.: On the Application of the Global Matched Filter to DOA Estimation with Uniform Circular Arrays. IEEE-T-SP 49, 702-709 (2001)

6. Van Trees, H.L.: Detection, Modulation and Estimation Theory, part 1. John Wiley and Sons, Chichester (1968)

7. Cox, D., Hinkley, D.: Theoretical Statistics. Chapman and Hall, Boca Raton (1974)

8. Fuchs, J.J.: Detection and estimation of superimposed signals. In: Proc. IEEE ICASSP, Seattle, vol. III, pp. 1649-1652 (1998)

9. Fletcher, R.: Practical Methods of Optimization. John Wiley and Sons, Chichester (1987)

10. Scharf, L.L.: Statistical signal processing, detection, estimation and time series analysis. Addison Wesley, Reading (1991)

11. Hotelling, H.: Tubes and spheres in n-spaces, and a class of statistical problems. Amer. J. Math. 61, 440-460 (1939)

12. Weyl, H.: On the volume of tubes. Amer. J. Math. 61, 461-472 (1939)

13. Fuchs, J.J.: On sparse representations in arbitrary redundant basis. IEEE-TIT 50(6), 1341-1344 (2004)

14. Villier, E., Vezzosi, G.: Caractéristiques opérationnelles de réception d'une classe de détecteurs. Gretsi, 15ème colloque, Juan les Pins, 125-128 (September 1995)

15. Fuchs, J.J.: A sparse representation criterion: recovery conditions and implementation issues. In: 17th World Congress IFAC, Seoul, July 2008, pp. 12425-12429 (2008)

16. Porat, B.: Digital Processing of Random Signals. Theory and Methods. Prentice Hall, New Jersey (1994) 